\title{
A quantum parametric oscillator with trapped ions
}

\author{
Shiqian Ding, ${ }^{1}$ Gleb Maslennikov, ${ }^{1}$ Roland Hablützel, ${ }^{1}$ Huanqian Loh, ${ }^{1}$ and Dzmitry Matsukevich ${ }^{1,2}$ \\ ${ }^{1}$ Centre for Quantum Technologies, National University of Singapore, 3 Science Dr 2, 117543, Singapore \\ ${ }^{2}$ Department of Physics, National University of Singapore, 2 Science Dr 3, 117551, Singapore
}

(Dated: December 8, 2015)

\begin{abstract}
A system of harmonic oscillators coupled via nonlinear interaction is a fundamental model in many branches of physics, from biophysics to electronics and condensed matter physics. In quantum optics, weak nonlinear interaction between light modes has enabled, for example, the preparation of squeezed states of light and generation of entangled photon pairs [1, 2]. While strong nonlinear interaction between the modes has been realized in circuit QED systems [3, 4], achieving significant interaction strength on the level of single quanta in other physical systems remains a challenge $[\underline{5}, 6$. Here we experimentally demonstrate such interaction that is equivalent to photon up- and downconversion using normal modes of motion in a system of two Yb ions 7, 8]. The nonlinearity is induced by the intrinsic anharmonicity of the Coulomb interaction between the ions and can be used to simulate fully quantum operation of a degenerate optical parametric oscillator [9]. We exploit this interaction to directly measure the parity and Wigner functions of ion motional states. The nonlinear coupling, combined with near perfect control of internal and motional states of trapped ions, can be applied to quantum computing [6, 10], quantum thermodynamics [11, 12], and even shed some light on the quantum information aspects of Hawking radiation [13].
\end{abstract}

Ions in a Paul trap experience a pseudopotential that is harmonic to a high degree and their motion is usually approximated by a set of normal modes that do not interact with each other. Coulomb interaction between the ions is, however, nonlinear and can introduce coupling between the modes and anharmonicity to the ion motion. The linear coupling of motional modes due to mutual Coulomb repulsion of ions was previously demonstrated in quantum regime [14, 15], where the ions were trapped in independent potential wells. The higher order terms in the Coulomb interaction lead, for example, to cross Kerr-type nonlinear coupling that results in a shift of the normal mode frequencies [7, 8] which has been experimentally observed [16].

In this Letter, we engineer the nonlinear interaction between modes of motion similar to a degenerate parametric oscillator at the single-phonon level. We consider a system of two ions with the same mass $m$ and charge $e$ in a linear Paul trap that is characterized by the singleion secular frequencies $\omega_{x}, \omega_{y}, \omega_{z}$ [7, 8]. The potential energy of the system has the form

$$
\begin{aligned}
V= & m \omega_{x}^{2}\left(X^{2}+x^{2}\right)+m \omega_{y}^{2}\left(Y^{2}+y^{2}\right) \\
& +m \omega_{z}^{2}\left(Z^{2}+z^{2}\right)+\frac{e^{2}}{8 \pi \epsilon_{0}} \frac{1}{\sqrt{x^{2}+y^{2}+z^{2}}},
\end{aligned}
$$

where $\epsilon_{0}$ is the permittivity of free space, $X, Y, Z$ are the center-of-mass coordinates, and $x, y, z$ are half the separation between the ions along the direction of principle trap axes.

When $\omega_{z}<\left(\omega_{x}, \omega_{y}\right)$, the ions crystallize along the axial $(z)$ direction at an equilibrium distance $z_{0}$ from the trap center. According to Eq. 1, the motion of the centerof-mass modes is harmonic, but the out-of-phase modes are coupled to each other by the Coulomb interaction. For small axial displacement $u=z-z_{0}$ and keeping only terms up to the third order that contribute to the coupling between the $x$ and $z$ modes, the potential energy becomes [8]

$$
V=m \omega_{r}^{2} x^{2}+m \omega_{s}^{2} u^{2}+\frac{m \omega_{s}^{2}}{z_{0}} x^{2} u+\ldots .
$$

Here $\omega_{s}=\sqrt{3} \omega_{z}, \omega_{r}=\sqrt{\omega_{x}^{2}-\omega_{z}^{2}}$ are the axial ("stretch") and radial ("rocking") mode frequencies for the out-of-phase motion. If the trap frequencies are chosen such that $\omega_{s} \simeq 2 \omega_{r}$, we can apply the standard transformations $\hat{x}=\left(\hbar / 4 m \omega_{r}\right)^{1 / 2}\left(\hat{a}+\hat{a}^{\dagger}\right), \hat{u}=$ $\left(\hbar / 4 m \omega_{s}\right)^{1 / 2}\left(\hat{c}+\hat{c}^{\dagger}\right)$ and express the Hamiltonian in the rotating wave approximation as

$$
\hat{H}=\hbar \omega_{r} \hat{a}^{\dagger} \hat{a}+\hbar \omega_{s} \hat{c}^{\dagger} \hat{c}+\hbar \xi\left(\hat{a}^{\dagger} \hat{c}+\hat{a}^{2} \hat{c}^{\dagger}\right),
$$

where $\hat{c}^{\dagger}, \hat{c},\left(\hat{a}^{\dagger}, \hat{a}\right)$ are the phonon creation and annihilation operators in axial (radial) mode. The first two terms in Eq. 3 describe harmonic motion in the axial (radial) mode with the frequency $\omega_{s}\left(\omega_{r}\right)$ and the third term couples these modes with the coupling coefficient given by

$$
\xi=\frac{1}{8 z_{0}} \sqrt{\frac{\hbar \omega_{s}^{3}}{m \omega_{r}^{2}}} .
$$

The coupling is nonlinear: one phonon from the axial mode is converted into a pair of phonons in the radial mode and vice versa.

A schematic of the experimental setup is shown in Fig. 1. Two ${ }^{171} \mathrm{Yb}^{+}$ions are confined in a four-rod linear rf-Paul trap with the single-ion secular frequencies $\left(\omega_{x}, \omega_{y}, \omega_{z}\right) / 2 \pi=(0.99,0.90,0.75) \mathrm{MHz}$. To change the detuning $\delta=\omega_{s}-2 \omega_{r}$, the axial trapping frequency remains fixed for all the experiments, while the radial frequency is tuned by adjusting the DC voltages applied to 

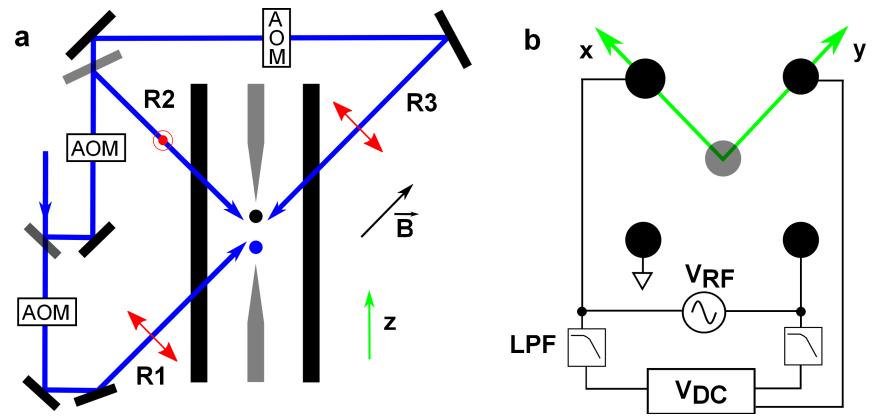

FIG. 1. Experimental setup. a. Schematic of the experimental setup. The two-ion crystal is aligned along the axial direction of the trap. One of the $\mathrm{Yb}$ ions is in the $S_{1 / 2}$ ground state and interacts with Doppler cooling and Raman beams. The other ion is prepared in a dark metastable ${ }^{2} F_{7 / 2}$ state and is sympathetically cooled. Three Raman beams are directed onto the ions in such a way that the motional states along the axial and radial directions are controlled by the R1-R2 pair and R2-R3 pair, respectively. The red labels show the polarizations of Raman beams. The 7.0 Gauss magnetic field $\vec{B}$ is parallel to the R1 Raman beam. b. Trap electrode configuration. The trap consists of two end-cap electrodes separated by about $2.0 \mathrm{~mm}$ and four rods. Each trap rod has a diameter of $0.5 \mathrm{~mm}$ and the center-to-center separation is $0.9 \mathrm{~mm}$. The axial trapping frequency is controlled by the DC voltages applied to the end caps, and the radial trapping potential is generated by $30 \mathrm{MHz}$ rf signal connected to the diametrically opposite rods in the $x$ direction. The fine control of radial motional frequencies is achieved by adjusting offset voltages on the $x$ electrodes with two pairs of low pass filters (LPF) with different time constants (see Methods). Small additional DC voltages applied to the $x$ and $y$ electrodes help to compensate stray electric fields.

the $x$ electrodes (see Fig. 1b). One of the ions is optically pumped into a metastable ${ }^{2} F_{7 / 2}$ state and does not interact with the laser beams during the experiment. At the beginning of every experimental sequence, the detuning is set at $\delta / 2 \pi=35 \mathrm{kHz}$, which is much larger than $\xi / 2 \pi$, effectively decoupling two modes. We then initialize all the motional modes of the two-ion crystal in the ground state by Doppler cooling followed by sideband cooling [17]. The spin-motion coupling for the sideband cooling is achieved by driving frequency-comb-assisted Raman transitions [18] (see Methods). The residual population $\bar{n}$ of all the motional modes after sideband cooling is well below 0.05 phonons.

To verify the nonlinearity of the coupling at the single phonon level, we initially populate the radial mode with either one or two phonons as described in Methods section. We then change DC voltages applied to the rods of the trap, with time constants of around $20 \mu \mathrm{s}$, to bring the detuning $\delta$ to zero. After time $\tau$, we bring the detuning back to its initial value and check for the presence of phonons in the axial or radial mode. The results are presented in Fig. 2,

We observe energy oscillations between the axial and

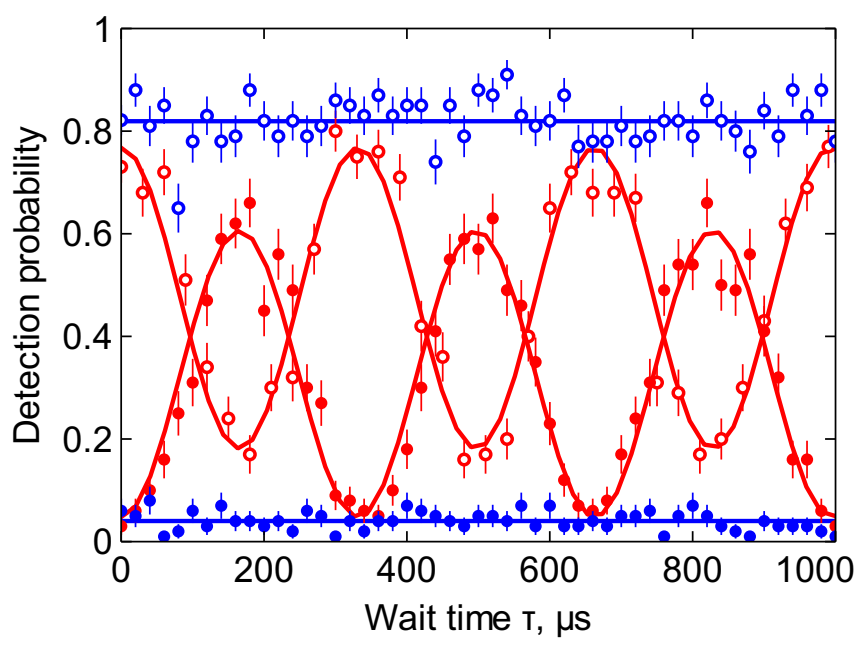

FIG. 2. Phonon state evolution in the axial (solid dots) and radial (open circles) modes, where either one (blue) or two (red) phonons is initially added to the radial mode. The sinusoidal fit (red line) reveals the coupling rate to be $3.02 \pm 0.02 \mathrm{kHz}$. The straight fit lines show that there is negligible coupling between the modes when only one radial phonon is present. The error bars are statistical uncertainties that correspond to one standard deviation $\sigma$. The oscillations amplitude after about $10 \mathrm{~ms}$ is a factor of $0.39(7)$ compared to the initial amplitude, limited by the coherence time of the phonons in the radial mode.

radial modes only when the radial mode is initially prepared in the two-phonon Fock state. The measured oscillation frequency $3.02 \pm 0.02 \mathrm{kHz}$ is compatible with the frequency $2 \sqrt{2} \xi / 2 \pi=2.96 \mathrm{kHz}$ predicted by Eq. 4. The reduced visibility of the oscillation together with the small deviation of the measured coupling strength from theory can be attributed to two sources: the non-perfect mapping of the motional state to the internal state of the ion, and the deviation from the resonance condition, compatible with the observed frequency drifts.

The observed mode coupling is analogous to the upand down-conversion of photons in nonlinear crystals. However, in contrast to the optical case, where the number of pump photons required to produce one photon pair is usually large [6], the coupling strength here is much higher and this effect can be readily observed even at the single quantum level.

In order to further quantify this coupling, we probe the avoided crossing of the coupled modes of motion. Without the coupling $(\xi \rightarrow 0)$, the bare energy eigenstates of the Hamiltonian in Eq. 3 are degenerate and cross when $\omega_{s}=2 \omega_{r}$. The coupling mixes bare energy eigenstates of the system such that the new eigenstates have nonzero projection along both the axial and radial directions. This results in the mode splitting that we measure in the vicinity of blue sideband of axial mode. The results are shown in Fig. 3 ,

If the detuning $\delta$ changes on a time scale much longer 


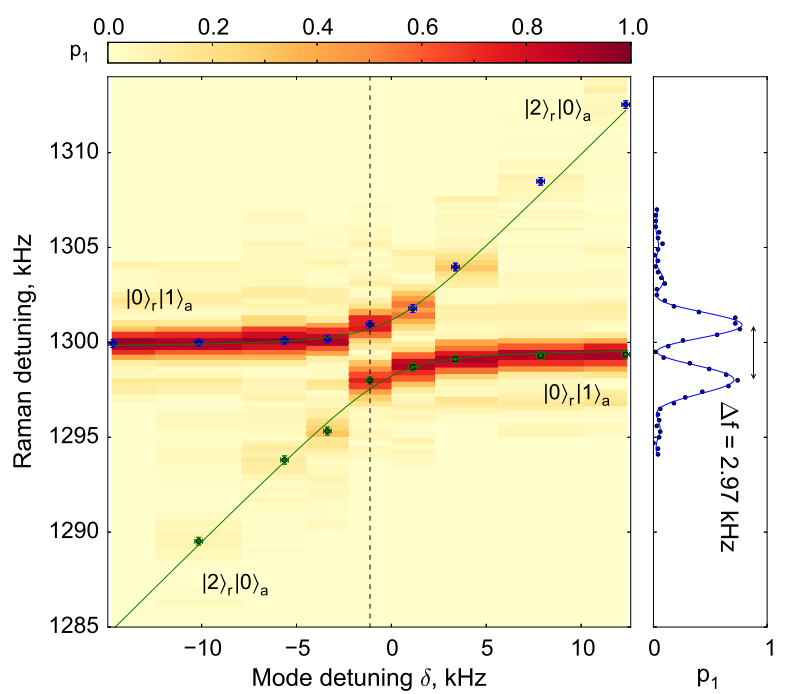

FIG. 3. Avoided crossing observed for the axial mode after sideband cooling. The left plot shows the probability $p_{1}$ of the transition from a "dark" $\left|F=0, m_{F}=0\right\rangle$ to "bright" $\left|F=1, m_{F}=0\right\rangle$ state as a function of the detuning $\delta$ from the resonance condition, and the Raman detuning $\Delta$. We extract the coupling strength by measuring the mode splitting at resonance, as shown on the right panel. The splitting is measured to be $2.97 \pm 0.03 \mathrm{kHz}$. Dots correspond to the measured frequencies at the peak centers and the solid lines show the eigenvalues of the Hamiltonian (3).

than the inverse coupling rate $2 \pi / \xi$, the system remains in the same energy eigenstate, leading to the adiabatic evolution of motional states between the radial and axial modes. In particular, the lowest energy eigenstate for $\omega_{s}>2 \omega_{r}$, i.e. $|n\rangle_{r}|0\rangle_{a}$, will evolve into the lowest energy eigenstate for $\omega_{s}<2 \omega_{r}$. The latter eigenstate is $|0\rangle_{r}|n / 2\rangle_{a}$ for even $n$ and $|1\rangle_{r}|(n-1) / 2\rangle_{a}$ for odd $n$. Therefore, the adiabatic sweep enables direct parity measurement of the ion motional state: the absence or presence of a phonon in the radial mode after the sweep provides information about the parity of the initial state of this mode. We detect the phonon by mapping it onto the ion internal state and then determine the expectation value of the parity operator $\hat{P}|n\rangle_{r}=(-1)^{n}|n\rangle_{r}$ as $\langle\hat{P}\rangle=\left(1-2 p_{1} / \eta\right)$, where $\eta=0.86$ is the phonon mapping efficiency (see Methods), and $p_{1}$ is the probability to find the ion in the "bright" internal state after mapping.

The direct parity measurement opens the door to efficiently determining the Wigner function of a quantum state [19]. It was shown in 20 22] that the Wigner function relates to the parity operator by 23 26

$$
W(\alpha)=\frac{2}{\pi} \operatorname{Tr}[D(-\alpha) \rho D(\alpha) \hat{P}],
$$

where $\rho$ is the density matrix and $D(\alpha)$ is the displacement operator. The state $D(-\alpha) \rho D(\alpha)$ corresponds to a displacement of the state $\rho$ by the amount $-\alpha$ in phase space. The displacement can be carried out by applying a force to the ion with controlled phase and duration. After an adiabatic sweep of the radial trapping frequency with a time constant of $2 \mathrm{~ms}$ (see Fig. 10), and mapping the radial phonon onto the internal state of the ion, the value of the Wigner function can be determined as $W(\alpha)=2\langle\hat{P}\rangle / \pi$.

The measurement protocol of the Wigner function was tested on several quantum states, prepared as described in the Methods section. Figures 4 a-f show the Wigner functions for the vacuum state, coherent states, and Schrödinger cat states. The measured Wigner functions are shown in the top row and can be compared with the corresponding theoretical predictions plotted beneath them. Figures $45-j$ show the Wigner functions for the Fock states with phonon number $n=1,2$ and 5 as a function of $|\alpha|$. Some discrepancy between the theory and experiment is likely due to anharmonicity of the motional mode that causes an amplitude-dependent rotation of the Wigner function about the origin.

In conclusion, we have demonstrated strong nonlinear coupling between the motional states of a two-ion crystal described by a Hamiltonian similar to degenerate optical parametric down-conversion. When combined with nearly deterministic phonon preparation and readout, both of which are readily available in an ion trap, the nonlinear coupling allows us to directly measure the parity of the ion motional state and determine its Wigner function. This nonlinearity can be harnessed as a tool to implement hybrid quantum computations that involve both discrete [6] and continuous variables [10], or to simulate a molecular BEC formation [27]. One can also extend the this type of coupling to three modes in a three-ion crystal 7], thereby realizing absorption refrigerators in the quantum regime [11], or even simulating quantum information aspects of Hawking radiation [13].

During preparation of this manuscript, we became aware of related work of Kienzler et al. 28], where a different method of the Wigner function reconstruction based on the measurements in a squeezed Fock basis was demonstrated.

We acknowledge discussions with Berthold-Georg Englert, Manas Mukherjee and Alex Kuzmich. This research was supported by the National Research Foundation and the Ministry of Education of Singapore.

\section{METHODS}

\section{Experimental setup}

The ions are Doppler cooled by a $369.53 \mathrm{~nm}$ laser of intensity $3 \mathrm{~W} / \mathrm{cm}^{2}$ and red-detuned from the ${ }^{2} S_{1 / 2}, F=$ $1 \rightarrow{ }^{2} P_{1 / 2}, F=0$ transition. A $935.19 \mathrm{~nm}$ laser of intensity $25 \mathrm{~W} / \mathrm{cm}^{2}$ repumps the population from the long-lived ${ }^{2} D_{3 / 2}$ state. A magnetic field of $7.0 \mathrm{G}$ applied along the propagation direction of the R1 Raman 

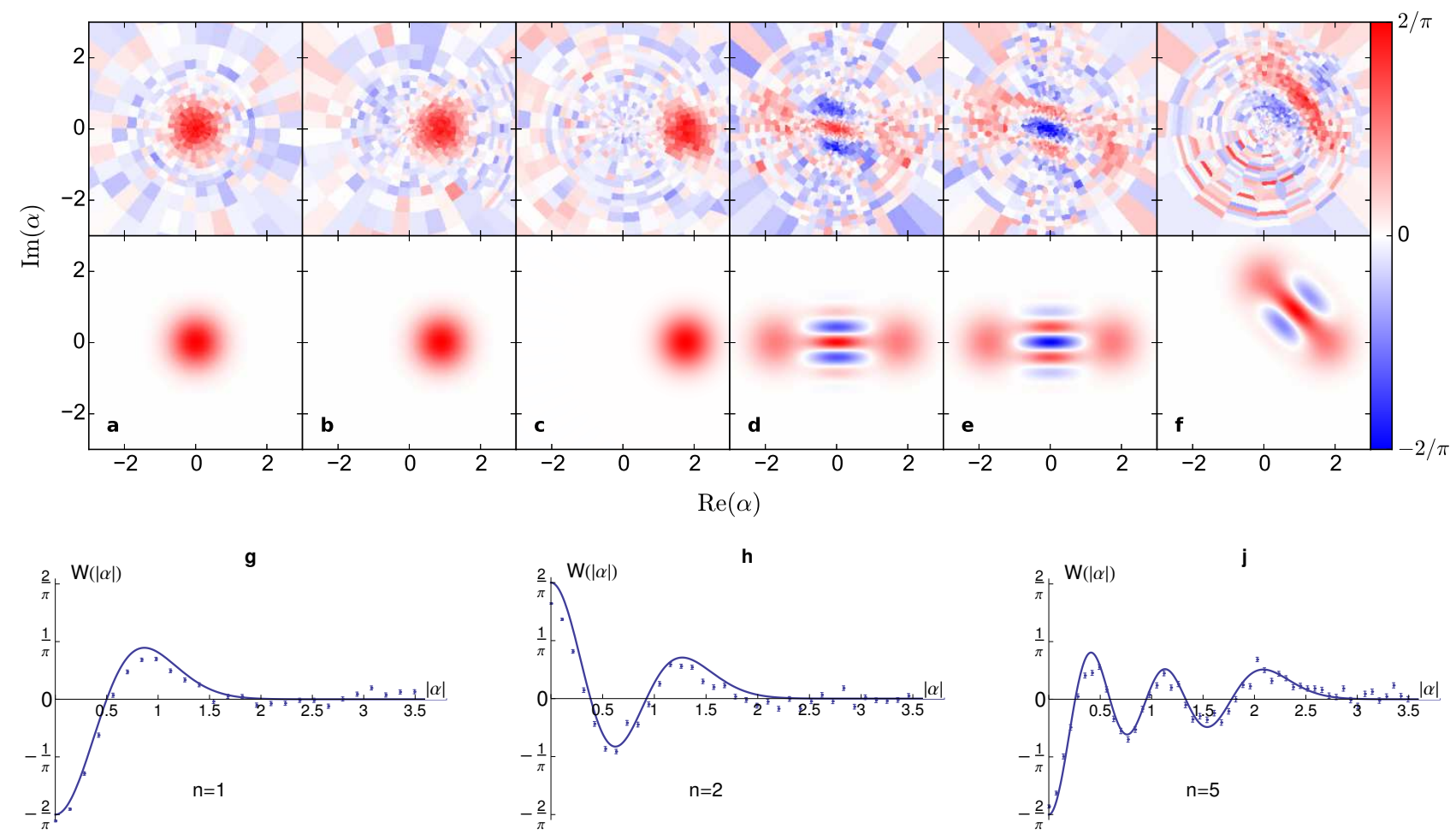

FIG. 4. Wigner functions for different quantum states. a-f. Wigner functions (from left to right) for the vacuum state, coherent states $|\alpha\rangle_{r}$ for $\alpha=0.87(3)$ and 1.73(6), Schrödinger cat states $\left(|\alpha\rangle_{r}+|-\alpha\rangle_{r}\right) / \sqrt{2},\left(|\alpha\rangle_{r}-|-\alpha\rangle_{r}\right) / \sqrt{2}$ and $\left(|\alpha\rangle_{r}-|i \alpha\rangle_{r}\right) / \sqrt{2}$ for $\alpha=1.73(6)$. The top row corresponds to the experimental data, while the bottom row shows the calculated Wigner functions. g-j. Wigner functions of the Fock states $|n\rangle_{r}$ averaged over the phase of $\alpha$ with $n=1,2$, and 5 . The solid lines show the theoretical prediction $W(|\alpha|)=2(-1)^{n} e^{-2|\alpha|^{2}} L_{n}\left(4|\alpha|^{2}\right) / \pi$; the points are experimentally measured values. The error bars show $1 \sigma$ statistical uncertainty. The negative measured values of the Wigner functions for the Schrödinger cat and Fock states demonstrate the non-classical character of these states.

beam defines the quantization axis and destabilizes the dark states in the ${ }^{2} S_{1 / 2}$ and ${ }^{2} D_{3 / 2}$ manifolds for efficient Doppler cooling. We use the standard optical pumping and resonance fluorescence state detection technique [29] to initialize and detect the ion's quantum state. The "dark" and "bright" states used in state detection are $|0\rangle \equiv\left|S_{1 / 2}, F=0, m_{F}=0\right\rangle$ and $|1\rangle \equiv \mid S_{1 / 2}, F=$ $\left.1, m_{F}=0\right\rangle$, respectively. One of the ions is pumped into a metastable ${ }^{2} F_{7 / 2}$ state with two photons, one from the $369 \mathrm{~nm}$ Doppler cooling laser and the other at $386.8 \mathrm{~nm}$ from a femtosecond modelocked pulsed laser that drives the ${ }^{2} P_{1 / 2} \rightarrow(7 / 2,2)_{3 / 2}$ transition.

The ponderomotive trapping potential is generated by applying a rf signal at $30 \mathrm{MHz}$ to two diametrically opposite rods of the trap (Fig. 1 $b$ ). The radial trapping frequency is actively stabilized using a signal from a pickup coil that is positioned outside the vacuum chamber at a distance of around $5 \mathrm{~cm}$ from the trap. The systematic drift of the radial trapping frequency $\omega_{x} / 2 \pi$ is less than $200 \mathrm{~Hz} /$ hour. The axial trapping frequency is independently controlled by DC voltages applied to the end caps and has negligible systematic drift.

We also measure the coherence time of the phonons in the axial and radial out-of-phase modes with a Ramsey experiment [16] to be 55(7) $\mathrm{ms}$ and 10.2(9) ms, respectively. The latter is limited by trapping frequency instability and can be extended to 38(4) ms in spin-echo type measurements.

\section{Raman beams}

The Raman beams are produced by a frequencydoubled mode-locked Ti:Sapphire laser (pulse duration $3 \mathrm{ps}$, repetition rate $76 \mathrm{MHz}$ ). The resulting beam has a central wavelength of $374 \mathrm{~nm}$ and an average power of $250 \mathrm{~mW}$. It is then split into three, sent through three acousto-optical modulators (AOMs), and focused to beam waists of around $15 \mu \mathrm{m}$ at the ions' position as shown in Fig. Th. The R1 and R2 beams form $45^{\circ}$ and $135^{\circ}$ angles, respectively, with the $z$-axis, while $\mathrm{R} 3$ is counter-propagating to R1. The path lengths of all three beams are matched to a precision much better than the 
picosecond pulse length. The polarizations of the R1 and R2 beams are linear and mutually orthogonal, and the R3 beam polarization is parallel to that of $\mathrm{R} 1$.

In this configuration, the R1-R2 (R2-R3) pair couples the axial (radial) motional mode to the internal state of the ion by driving stimulated Raman transitions [17, 18]. By adjusting the Raman detuning, we may drive the "carrier" $(|0\rangle|n\rangle \rightarrow|1\rangle|n\rangle)$, the "red" $(|0\rangle|n\rangle \rightarrow|1\rangle|n-1\rangle)$ or "blue" $(|0\rangle|n\rangle \rightarrow|1\rangle|n+1\rangle)$ sideband transitions. Here the first ket state corresponds to ion internal state, and the second to the state of its motional mode.

Moreover, when the R1 and R2 (R2 and R3) beams are detuned from each other, the polarization gradient of the resulting optical lattice at the location of the ion oscillates. This leads to a periodic optical dipole force applied to the ion in the state $|2\rangle \equiv \mid S_{1 / 2}, F=1, m_{F}=$ $1\rangle$. The ion in the state $|0\rangle$ does not experience the force because its Stark shift is independent of polarization [30].

\section{Control of normal mode frequencies}

The axial trapping frequency is controlled by DC voltages applied to the end caps, and the radial trapping potential is generated by $30 \mathrm{MHz}$ rf signal connected to the diametrically opposite rods in the $x$ direction. The radial trapping frequencies are shifted by DC offset voltages on the level of hundreds of millivolts applied to the $x$ electrodes such that only the radial mode along the $x$ direction interacts with the axial mode, while the radial mode along the $y$ direction is far off resonance $(|\delta| / 2 \pi>200 \mathrm{kHz})$. The radial trapping frequency $\omega_{r}$ can be slowly changed between two values with the help of two identical low-pass RC filters (LPF) that have a time constant of $2 \mathrm{~ms}$, or rapidly changed with a time constant of $20 \mu \mathrm{s}$ using different pairs of filters.

The fast time scale $(20 \mu \mathrm{s})$ is much shorter than the inverse coupling strength $2 \pi / \xi$, while the slow time scale ( $2 \mathrm{~ms}$ ) is much longer than $2 \pi / \xi$ to satisfy the adiabaticity criterion. Both timescales are much larger than one oscillation period of the ion crystal, and we have experimentally verified that no significant motional excitations are induced during these frequency sweeps [31, 32].

\section{Phonon mapping efficiency}

In our setup, the mapping of the phonon to the internal state of the ion is achieved by applying a $\pi$-pulse on the red sideband that simultaneously removes one phonon from the motional mode and changes the internal state of the ion. This procedure is not perfect, and limited by the residual population of the other motional modes, power stability of the Raman lasers and the internal state detection efficiency. To satisfy the condition $W(\alpha) \rightarrow 0$ for large $\alpha$, we determine the phonon mapping efficiency to be $\eta=0.86 \pm 0.01$. This measurement agrees well with the result of an alternative method, where the radial mode is initialized as a single-phonon Fock state and the probability of making a spin flip is subsequently found to be $\eta=0.89 \pm 0.04$.

\section{Preparation of quantum states}

To prepare the coherent states shown in Fig. 4b,c, we start with all the motional modes of the ion crystal cooled to the ground state (Fig. 4 a) and the $S_{1 / 2}$ ion pumped to the state $|0\rangle$. A microwave $\pi$-pulse drives the transition $|0\rangle \rightarrow|2\rangle$. The optical lattice, formed by the R2 and R3 beams and running at the frequency $\omega_{r}$, applies a force to coherently excite the ion motion 30.

The displacement in phase space $\alpha$ is calibrated by measuring the average number of phonons $\bar{n}$ in the radial mode as a function of the coherent excitation duration. The measurement yields $|\alpha|^{2}=\bar{n}=3.0(2) * 10^{-4} * t^{2}$, where $t$ is expressed in microseconds.

To prepare the Schrödinger cat states shown in Fig 4d$\mathrm{f}$, we follow the method similar to [33]. We prepare the $S_{1 / 2}$ ion in the state $(|0\rangle+|2\rangle)|0\rangle_{r} / \sqrt{2}$ by the microwave $\pi / 2$ pulse and then apply a spin-dependent optical dipole force on resonance with the radial mode of motion that only displaces the ion in the internal state $|2\rangle$ [30]. After that, we swap the internal states of the ions with a microwave $\pi$-pulse and apply the force with the phase shift $\phi$ to produce the $\left(|0\rangle|\alpha\rangle_{r}+|2\rangle\left|\alpha e^{i \phi}\right\rangle_{r}\right) / \sqrt{2}$ state. Finally, we apply a microwave $\pm \pi / 2$ - pulse to the internal state of the ion and arrive at the state $|0\rangle\left(|\alpha\rangle_{r} \pm\left|\alpha e^{i \phi}\right\rangle_{r}\right) / 2+|2\rangle\left(|\alpha\rangle_{r} \mp\left|\alpha e^{i \phi}\right\rangle_{r}\right) / 2$. We then detect the internal state of the ion using the standard fluorescence technique. If the ion is found in the $|0\rangle$ internal state, the ion has scattered no photons and the motional state of the ions is projected onto the Schrödinger cat state $\left(|\alpha\rangle_{r} \pm\left|\alpha e^{i \phi}\right\rangle_{r}\right) / \sqrt{2}$. If the ion is found in the "bright" state $|2\rangle$, the motional state is destroyed by the photon recoil and we omit these cases.

The Fock states shown in Fig. 45 -j are generated by the following sequence: a $\pi$-pulse on the blue sideband of the $|0\rangle \rightarrow|1\rangle$ transition that adds a phonon to the motional mode, followed, if necessary, by another $\pi$-pulse on the red sideband that adds another phonon, or a $\pi$ pulse on the carrier transition that returns the ion to the initial internal state. To generate the $n$-phonon Fock state $|n\rangle_{r}$, $n$ sideband pulses are applied.

[1] Kwiat, P. G. et al. New high-intensity source of polarization-entangled photon pairs. Phys. Rev. Lett. 75, 4337-4341 (1995).

[2] Wu, L.-A., Kimble, H. J., Hall, J. L. \& Wu, H. Gener- 
ation of squeezed states by parametric down conversion. Phys. Rev. Lett. 57, 2520-2523 (1986).

[3] Lecocq, F. et al. Coherent frequency conversion in a superconducting artificial atom with two internal degrees of freedom. Phys. Rev. Lett. 108, 107001 (2012).

[4] Holland, E. T. et al. Single-photon-resolved cross-kerr interaction for autonomous stabilization of photon-number states. Phys. Rev. Lett. 115, 180501 (2015).

[5] Feizpour, A., Hallaji, M., Dmochowski, G. \& Steinberg, A. M. Observation of the nonlinear phase shift due to single post-selected photons. Nat Phys 11, 905-909 (2015).

[6] Langford, N. K. et al. Efficient quantum computing using coherent photon conversion. Nature 478, 360-363 (2011).

[7] Marquet, C., Schmidt-Kaler, F. \& James, D. Phononphonon interactions due to non-linear effects in a linear ion trap. Applied Physics B 76, 199-208 (2003).

[8] Nie, X., Roos, C. F. \& James, D. Theory of cross phase modulation for the vibrational modes of trapped ions. Physics Letters A 373, 422 - 425 (2009).

[9] Mandel, L. \& Wolf, E. Optical Coherence and Quantum Optics (Cambridge University Press, Cambridge, U.K., 1995).

[10] Andersen, U. L., Neergaard-Nielsen, J. S., van Loock, P. \& Furusawa, A. Hybrid discrete- and continuous-variable quantum information. Nat Phys 11, 713-719 (2015).

[11] Correa, L. A., Palao, J. P., Alonso, D. \& Adesso, G. Quantum-enhanced absorption refrigerators. Sci. Rep. 4 (2014).

[12] Roßnagel, J., Abah, O., Schmidt-Kaler, F., Singer, K. \& Lutz, E. Nanoscale heat engine beyond the carnot limit. Phys. Rev. Lett. 112, 030602 (2014).

[13] Nation, P. D. \& Blencowe, M. P. The trilinear hamiltonian: a zero-dimensional model of hawking radiation from a quantized source. New Journal of Physics 12, 095013 (2010).

[14] Brown, K. R. et al. Coupled quantized mechanical oscillators. Nature 471, 196-199 (2011).

[15] Harlander, M., Lechner, R., Brownnutt, M., Blatt, R. \& Hansel, W. Trapped-ion antennae for the transmission of quantum information. Nature 471, 200-203 (2011).

[16] Roos, C. F. et al. Nonlinear coupling of continuous variables at the single quantum level. Phys. Rev. A 77, 040302 (2008).

[17] Monroe, C. et al. Resolved-sideband raman cooling of a bound atom to the $3 \mathrm{~d}$ zero-point energy. Phys. Rev. Lett.
75, 4011-4014 (1995).

[18] Hayes, D. et al. Entanglement of atomic qubits using an optical frequency comb. Phys. Rev. Lett. 104, 140501 (2010).

[19] Wigner, E. On the quantum correction for thermodynamic equilibrium. Phys. Rev. 40, 749-759 (1932).

[20] Royer, A. Wigner function as the expectation value of a parity operator. Phys. Rev. A 15, 449-450 (1977).

[21] Englert, B.-G., Sterpi, N. \& Walther, H. Parity states in the one-atom maser. Optics Communications 100, 526 535 (1993).

[22] Lutterbach, L. G. \& Davidovich, L. Method for direct measurement of the wigner function in cavity qed and ion traps. Phys. Rev. Lett. 78, 2547-2550 (1997).

[23] Bertet, P. et al. Direct measurement of the wigner function of a one-photon fock state in a cavity. Phys. Rev. Lett. 89, 200402 (2002).

[24] Deleglise, S. et al. Reconstruction of non-classical cavity field states with snapshots of their decoherence. Nature 455, 510-514 (2008).

[25] Sun, L. et al. Tracking photon jumps with repeated quantum non-demolition parity measurements. Nature 511, 444-448 (2014).

[26] Leibfried, D. et al. Experimental determination of the motional quantum state of a trapped atom. Phys. Rev. Lett. 77, 4281-4285 (1996).

[27] Jin, G.-R., Kim, C. K. \& Nahm, K. Quantum dynamics and statistical properties of atom-molecule bose-einstein condensates. Phys. Rev. A 72, 045602 (2005).

[28] Kienzler, D. et al. Observation of quantum interference between separated mechanical oscillator wavepackets. submitted (2015).

[29] Olmschenk, S. et al. Phys. Rev. A 76, 052314 (2007).

[30] Ding, S. et al. Microwave control of trapped-ion motion assisted by a running optical lattice. Phys. Rev. Lett. 113, 073002 (2014).

[31] Poulsen, G. \& Drewsen, M. Adiabatic cooling of a single trapped ion. arXiv preprint arXiv:1210.4309 (2012).

[32] Noguchi, A., Shikano, Y., Toyoda, K. \& Urabe, S. Aharonov-bohm effect in the tunnelling of a quantum rotor in a linear paul trap. Nature communications $\mathbf{5}$ (2014).

[33] Monroe, C., Meekhof, D. M., King, B. E. \& Wineland, D. J. A schrodinger cat superposition state of an atom. Science 272, 1131-1136 (1996). 